\title{
Comparative nutritional studies of sheep and goats fed cultivated tree legumes mixture under desert condition
}

\author{
Helal H. G., Nassar M. S., Badawy H. S., Eid E.Y and El Shaer H. M. \\ Animal and Poultry Nutrition Department Desert Research Center, P.O. Code 11753 Mataria, Cairo, Egypt.
}

Received 12 January 2018; Accepted 1 April 2018; Published Online 28 April 2018

\author{
Address for Correspondence: \\ Helal, H.G. Associate Professor, Animal and Poultry nutrition Department, Animal and Poultry Production Division, Desert Research \\ Center, Mataria, Cairo, Egypt.
}

E-mail: godahassan@yahoo.com

Copyright () 2018 by authors and American-Eurasian Network for Scientific Information.

This work is licensed under the Creative Commons Attribution International License (CC BY).

http://creativecommons.org/licenses/by/4.0/

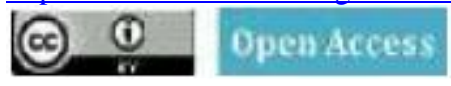

\begin{abstract}
This research was performed to compare the nutritional studies of Barki sheep and doe Shami goats fed on mixture of forage tree legumes and its effects on feed intake, nutrient digestibility, nutritive value, nitrogen utilization, body weight change, some parameters of rumen and blood. Our study was carried out at South Sinai Research Station (Ras Suder), South Sinai Governorate, Egypt. Thirty-six pregnant Barki sheep and Shami goats were randomly allocated into two equal groups (18 animals each specie). The first group (G1) of each species fed alfalfa with concentrate diet and served as control. The second group (G2) fed ad libtum sun-dried chopped mixture contained 50\% Acacia saligna, 25\% Prosopis juliflora and $25 \%$ Leucaena leucocephala with concentrate diet. Results indicated that overall roughage and total DM intake (g/Kg $\left.\mathrm{W}^{0.75} / \mathrm{d}\right)$ were similar between both species but animals fed G2 showed lower $(\mathrm{P}<0.05)$ in roughage and total DM intake than those fed $\mathrm{G} 1$. The results also showed that goats had higher $(\mathrm{P}<0.05)$ overall dry matter $(\mathrm{DM})$, crud protein $(\mathrm{CP})$ and crud fiber $(\mathrm{CF})$ digestibility than sheep but overall nitrogen intake (NI) was similar between both species and was higher $(\mathrm{P}<0.05)$ for animals fed G1 vs. those fed G2. Also, nitrogen balance was similar between both species and was insignificant higher for those fed G1 vs. G2. Goats had higher $(\mathrm{P}<0.05)$ overall ammonia NH3-N than sheep but sheep had higher $(\mathrm{P}<0.05)$ overall ruminal TVFA's than goats as well as animals fed G2 showed lower $(\mathrm{P}<0.05)$ NH3$\mathrm{N}$ and TVFA's than those fed G1. Values of body weight change $(\mathrm{kg})$ and relative body weight $(\%)$ of end pregnancy were insignificant between both species and feeding treatments. Sheep had slightly insignificant lower concentrations of overall total protein and globulin than goats and was also slightly insignificant higher concentrations of overall urea and creatinine than goats while goats recorded insignificant higher values of overall AST and ALT than sheep. It was concluded feed utilization of tree legumes mixture as animal feeds in saline affected and soils could be an appropriate option for alleviating the desertification difficulties and provide alternatives as good feed resources especially in droughts when the other traditional food resources are shortage.
\end{abstract}

KEY WORDS:

Sheep, goats, tree legumes, intake, digestibility, rumen parameters and blood parameters.

\section{INTRODUCTION}

Semi-desert climate of Egypt is characterized by hot summer, dry and mild winters, low rainfall (5-200 $\mathrm{mm} / \mathrm{a})$ and high evaporation rates $(1,500-2,400 \mathrm{~mm} / \mathrm{a})$. The country is characterized by particularly good wind systems with excellent locations along the shores of the Red and Mediterranean Sea [1]. South Sinai is an arid region that affects excessive salt natural resources (water, soil, plants, etc.). Feeding trees, shrubs and agricultural by-products are important in animal production because they do not compete with human food and can provide significant food supplements, especially in the dry season [2]. The use of forage trees and shrubs to solve problems associated with low productivity in small ruminant production has received research attention in recent years [3]. Smallholder ruminant farmers in developing countries cannot afford concentrates and rely almost entirely on feed browse fodders to replenish their stocks. Acacia (Acacia saligna) is a leguminous shrub which provides large amounts of fodder for ruminants in marginal regions [4, 5]. A. saligna has reasonably 
large amount of crude protein [6], high concentration of tannins [7, 8]. These compounds have adverse effect on the nutritional value to the browse species and also affect feed intake and digestibility [9]. Prosopis (Prosopis juliflora) is a leguminous shrub or tree that grows in semi-arid areas all over the world [10]. Also, prosopis has been reported to feed livestock by previous findings [11, 12, 13]. Moreover, [14] reported successful inclusion of prosopis at $30 \%$ level in the composite feed cattle without any negative influence on feed intake, digestibility and rumen fermentation. Leucaena (Leucaena leucocephala) a local available tree legume is used as hay, silage or fresh for ruminants due to its good characteristics such as elevated protein, palatability, vitamin and mineral especially sulfur content which positively affects microbial populations [15]. Moreover, leucaena has high digestibility of protein and dry mater [16]. However, [17] showed decreased dry matter intake, digestibility, protozoal populations and animal performance when feeding on leucaena, which contains some anti-nutrients factors such as phenolic compounds. Feeding a mixture of these tree legumes could be reduce and overcome the problems of palatability and toxic effects $[18,19,3]$. Accordingly, the objective of the study was to compare the nutritional performance of Barki sheep and doe Shami goats fed on a sun-dried chopped mixture of A. saligna, P. juliflora and L. leucocephala under arid and saline conditions of Southern Sinai in Egypt.

\section{MATERIALS AND METHODS}

The current study was carried out at Ras Suder Research Station which belongs to Desert Research Center, South Sinai Governorate, Egypt. This experiment aimed at investigate the effects of feeding mixture of acacia, prosopis and leucaena on feed intake, digestibility, nutritive value, body weight change, rumen and blood parameters of Barki sheep and doe Shami goats.

Preparation of fodder shrubs:

Three fodder trees legumes were collected with heights of approximately $1.0-1.5 \mathrm{~m}$. and diameters $(0.5-2.5$ $\mathrm{cm}$.) from the Farm of Ras Suder Research Station and mechanically chopped into small pieces before introducing to animals.

\section{Experimental animals and treatments:}

Thirty-six pregnant Barki sheep and Shami goats were randomly divided into two equal groups, eighteen Barki sheep an averaged $(26.56 \pm 0.61 \mathrm{~kg})$ body weight and eighteen Shami goats an averaged $(25.46 \pm 1.40)$ body weight. Nine animals of each species were used as control group and fed alfalfa hay (Medicago sativa L.) with concentrate feed mixture. The other nine animals were fed ad libtum sun-dried chopped mixture contained 50\% Acacia saligna, 25\% Prosopis juliflora and 25\% Leucaena leucocephala with concentrate diet. Diets were fed to cover total requirements of ewes and do as recommended by [20]. The chemical composition of mixture A. saligna, P. juliflora and L. leucocephala (Table 1) was determined according to [21].

Table 1: Chemical composition of experimental feed (as \% on DM basis).

\begin{tabular}{|l|c|c|c|c|c|c|c|}
\hline Item & DM & OM & CP & EE & CF & NFE & Ash \\
\hline Alfalfa hay & 92.77 & 85.18 & 15.54 & 2.53 & 22.63 & 44.48 & 14.82 \\
\hline Prosopis juliflora & 94.50 & 92.63 & 12.38 & 2.15 & 27.55 & 50.55 & 7.37 \\
\hline Acacia saligna & 92.74 & 92.44 & 11.72 & 1.45 & 30.86 & 48.41 & 7.56 \\
\hline Leucaena leucocephala & 93.66 & 91.96 & 13.63 & 2.25 & 24.53 & 51.55 & 8.04 \\
\hline *Tree legumes mixture & 92.61 & 92.51 & 13.06 & 2.04 & 28.28 & 49.13 & 7.49 \\
\hline Concentrate feed mixture & 93.75 & 89.20 & 14.72 & 3.15 & 12.27 & 59.06 & 10.80 \\
\hline
\end{tabular}

Dry matter: (DM), crude protein: $(\mathrm{CP})$, crude fiber: $(\mathrm{CF})$, ether extract: (EE), nitrogen free extract: (NFE), organic matter: $(\mathrm{OM})$.

\section{Management:}

Animals were housed indoors inside semi-closed pens for 2-month then moved to metabolic cages. Daily feed offered and refusals were collected and weighed for each group of animals to estimate the actual feed intake and fresh water consumption was provided to animals twice daily over the trial period. However, the biweekly body weight changes were calculated by subtracting the live body weight at the start of the trial period from the live body weight and the finish of the trial period within each group.

\section{Digestibility trails:}

Four animals from each group were randomly chosen and used in digestibility trial to determine nutrients digestibility, nutritive value and nitrogen balance. Animals were placed in metabolic cages, weighted at the beginning and the end of the digestibility trial that lasted for 15 days of which the first 8 days were considered for adaptation period, followed by another seven days as collection period. During digestibility trial periods, feed amounts and residuals were daily weighed and recorded. Fecal output and urine were daily collected each from each animal and kept for later analysis. Ten percent of each fecal sample was taken and dried at $65^{\circ} \mathrm{C}$ for a constant weight and ground to pass through a $1.0 \mathrm{~mm}$ mesh screen for chemical composition. At the end of the 
trial, samples of rumen liquid were taken place after 3 hours feeding to estimate rumen ammonia and volatile fatty acids.

Chemical analysis:

Dry matter (DM) content of feeds and feces determined by drying at $105^{\circ} \mathrm{C}$ for 24 hours, and organic matter $(\mathrm{OM})$ by ashing at $550^{\circ} \mathrm{C}$ in a muffle furnace for six hours. Ether extract (EE) and crude protein $(\mathrm{CP})$ were determined according to [21]. Rumen volatile fatty acids (TVFA's) were determined according to [22], while rumen ammonia nitrogen (NH3-N) was determined according to [21]. A total protein (TP) was determined according to Biuret method after [23]. On the other hand, albumin was determined according to [24]. Values of globulin were calculated by subtracting the value of albumin from the total protein whereas A/G ratio was calculated according to results of albumin and globulin. Total lipids concentration was determined according to [25]. Total cholesterol was carried out according to [26]. Glucose concentration was analyzed according to [27]. Concentrations of both alanine (ALT) and aspartate (AST) amino transferases were analyzed according to [28]. Alkaline phosphatase concentration (ALP) was determined according to [29]. Plasma urea and creatinine concentrations as indicators for kidney function were determined using bio diagnostic kits according to [30] and [31], respectively.

\section{Statistical analysis:}

All data were analyzed by the GLM procedure of SAS [32] with a model consisting of animal species $\times$ roughage group. Means were separated by least significant difference with a protected $\mathrm{F}$-test $(\mathrm{P}<0.05)$. Means were presented in tables for animal species $\times$ roughage group regardless of the significance of the interaction effect. Where the following model was used:

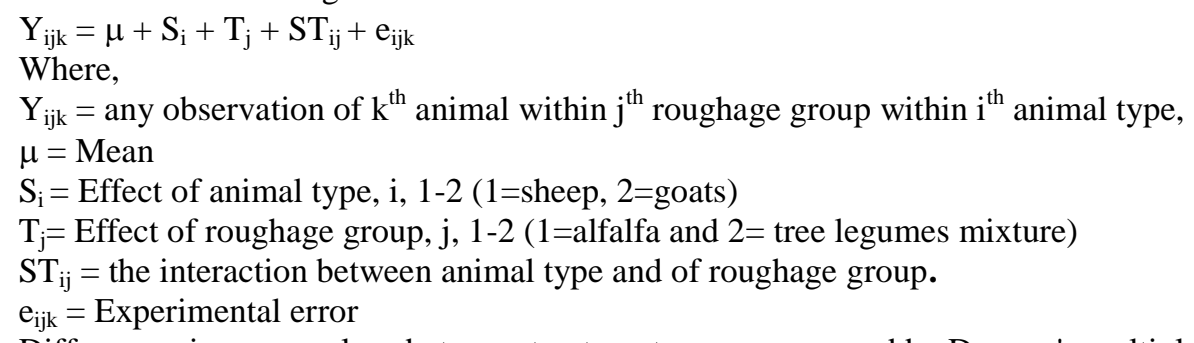

Differences in mean values between treatments were compared by Duncan's multiple range test [33].

\section{RESULTS AND DISCUSION}

\section{Chemical composition:}

Chemical compositions of the alfalfa hay, A. saligna, P. juliflora and L. leucocephala are presented in Table 1. The results showed that CP, EE and ash contents were higher in alfalfa hay than tree legumes mixture, while this mixture had higher $\mathrm{CP}, \mathrm{CF}, \mathrm{NFE}$ and OM\% which could be safe cover the important nutrients requirements for animals [3]. Also [34] suggest that browse with potential as nitrogen supplements for ruminants fed low quality fodders through dry season in semi-arid regions. Moreover, shrubs and multipurpose trees has become a useful alternative ruminant feed in harsh semi-arid region $[35,36]$. In addition, different studies suggested that the differences in their nutrient of forage legumes specially, CP and fiber content between these browse plants are probably due to sampling site, stage of plant growth and type (i.e., twigs, leaves or soft stem) of foliage sampled, season of collection, climatic influences soil type, fertility and water supply affect on nutrient concentrations in foliage growth [37, 38, 34].

\section{Feed intake:}

Mean values of body weight (BW), concentrate, roughage and total DM intake $\left(\mathrm{g} / \mathrm{Kg} \mathrm{W}{ }^{0.75} / \mathrm{d}\right)$ by compared sheep and goats are presented in Table 2. Average body weight $\left(\mathrm{Kg} \mathrm{W}^{0.75}\right)$ was insignificantly higher in sheep than goats and between feeding treatments. At similar trend was observed with overall concentrate intake $(\mathrm{g} / \mathrm{Kg}$ $\left.\mathrm{W}^{0.75} / \mathrm{d}\right)$ for both species and between feeding groups, while overall roughage and total DM intake $(\mathrm{g} / \mathrm{Kg} \mathrm{W}$ $0.75 / \mathrm{d})$ were similar between both species. However, animals fed G2 showed lower $(\mathrm{P}<0.05)$ in roughage and total DM intake $(\mathrm{g} / \mathrm{Kg} \mathrm{W}$ 0.75/d) than those fed G1. This agrees with the reports of [7] who noted that DMI/kg $\mathrm{W}^{0.75}$ was similar between sheep and goats when fed on Acacia cyanophylla as based diets (84.9 vs. 84.4, respectively). In many ways, researcher related differences in DMI in species and report that sheep consumed more DMI vs. goats $[39,34]$. Other studies reported that goats often eat more of some shrubs compere's sheep $[40,41,42]$. The lower values of dry roughage intake might be attributing to high content of anti-nutritional factors such as tannins [43]. Moreover, [44] reported that intake of mixed diets is an efficient way of diluting the adverse effects of secondary compounds in plants, such as tannins. 
Table 2: Mean values of dry matter intake of ewes and does as affected by feeding tree legumes mixture during digestibility trial.

\begin{tabular}{|c|c|c|c|c|}
\hline Item & Group & Sheep & Goats & Overall \\
\hline Average $\mathrm{BW}, \mathrm{Kg} \mathrm{W}^{0.75}$ & $\begin{array}{l}\text { G1 } \\
\text { G2 }\end{array}$ & $\begin{array}{l}14.94 \pm 0.37 \\
13.87 \pm 0.06\end{array}$ & $\begin{array}{l}12.81 \pm 0.85 \\
12.78 \pm 1.26\end{array}$ & $\begin{array}{l}13.88 \pm 0.59 \\
13.33 \pm 0.62\end{array}$ \\
\hline Overall & & $14.41 \pm 0.27$ & $12.80 \pm 0.70$ & $13.61 \pm 0.41$ \\
\hline Concentrate intake, $\mathrm{g} / \mathrm{Kg} \mathrm{W}$. $0.75 / \mathrm{d}$ & $\begin{array}{l}\text { G1 } \\
\text { G2 }\end{array}$ & $\begin{array}{l}34.90 \pm 1.21 \\
35.37 \pm 0.36\end{array}$ & $\begin{array}{l}35.17 \pm 1.55 \\
36.11 \pm 1.63\end{array}$ & $\begin{array}{l}35.04 \pm 0.91 \\
35.74 \pm 0.79\end{array}$ \\
\hline Overall & & $35.14 \pm 0.59$ & $35.64 \pm 1.06$ & $35.39 \pm 0.59$ \\
\hline Roughage intake, $\mathrm{g} / \mathrm{Kg} \mathrm{W}$. $0.75 / \mathrm{d}$ & $\begin{array}{l}\text { G1 } \\
\text { G2 }\end{array}$ & $\begin{array}{l}74.05^{\mathrm{a}} \pm 2.43 \\
35.41^{\mathrm{b}} \pm 6.81\end{array}$ & $\begin{array}{l}73.62^{\mathrm{a}} \pm 6.67 \\
32.75^{\mathrm{b}} \pm 3.59\end{array}$ & $\begin{array}{l}73.84^{\mathrm{a}} \pm 3.29 \\
34.08^{\mathrm{b}} \pm 3.60\end{array}$ \\
\hline Overall & & $54.73 \pm 8.03$ & $53.19 \pm 8.48$ & $53.96 \pm 5.65$ \\
\hline Total DM intake, $\mathrm{g} / \mathrm{Kg} \mathrm{W}^{0.75} / \mathrm{d}$ & $\begin{array}{l}\text { G1 } \\
\text { G2 }\end{array}$ & $\begin{aligned} 108.95^{\mathrm{a}} & \pm 3.04 \\
70.78^{\mathrm{b}} & \pm 6.95\end{aligned}$ & $\begin{array}{r}108.79^{\mathrm{a}} \pm 7.83 \\
68.86^{\mathrm{b}} \pm 4.42\end{array}$ & $\begin{array}{r}108.87^{\mathrm{a}} \pm 3.89 \\
69.82^{\mathrm{b}} \pm 3.83\end{array}$ \\
\hline Overall & & $89.87 \pm 8.02$ & $88.83 \pm 8.62$ & $89.35 \pm 5.69$ \\
\hline
\end{tabular}

G1, animals fed on alfalfa + CFM (control group).

$\mathrm{G} 2$, animals fed on a mixture of $A$. saligna $(50 \%)+P$. juliflora $(25 \%)+$ L. leucocephala $(25 \%)+\mathrm{CFM}$.

$\mathrm{a}, \mathrm{b}=$ values in the same column or row within a certain trait with different super scripts are significantly differed $(\mathrm{P}<0.05)$.

\section{Nutrient digestibility:}

Results of dry matter (DM), organic matter (OM), crude protein $(\mathrm{CP})$, crude fiber $(\mathrm{CF})$, either extract (EE) digestibility or nutritive values where shown in Table 3 . Goats had higher $(\mathrm{P}<0.05)$ digestibility coefficients of overall DM, CP and CF than sheep (71.93 vs. 67.83, 80.57 vs. 76.78 and 65.84 vs. $58.60 \%$, respectively) but there were insignificant differences among animal species in overall OM, EE and NFE digestibility. These results are in agreement with earlier reports by [34,45] whose reported that DM and CF digestibility were higher for in goats than sheep probably reflecting their better capacity to detoxify secondary compounds in the rumen goats vs. sheep [46]. Moreover, higher digestibility of nutrients by goats may relate to many reasons like greater number of total protozoa in the rumen, total gut length in goats is lower and the retention time of digesta in goats is higher than sheep [47]. On the other hand, animals fed G1 showed higher $(\mathrm{P}<0.05)$ overall DM, OM and NFE digestibility than those fed G2 (73.21 vs. $66.55,74.12$ vs. 68.21 and 77.56 vs. $68.61 \%$, respectively) but overall CP, CF and EE digestibility were similar between both treatments. The lower values in digestibility of tree legumes mixture may be attributed to high content of tannins [48, 49]. Tannins could can be reduce digestibility of crude protein and carbohydrate by inhibiting digestive enzymes and by altering permeability of the gut wall $[50,51]$. Results of nutritive values Table (3) indicated that animals fed G1 had higher $(\mathrm{P}<0.05)$ TDN and DCP intake $\left(\mathrm{g} / \mathrm{kg} \mathrm{W}^{0.75}\right)$ than those fed $\mathrm{G} 2$ but overall TDN and DCP intakes $\left(\mathrm{g} / \mathrm{kg} \mathrm{W}^{0.75}\right)$ were similar between both species.

Table 3: Mean values of digestion coefficients by ewes and does as affected by feeding tree legumes mixture.

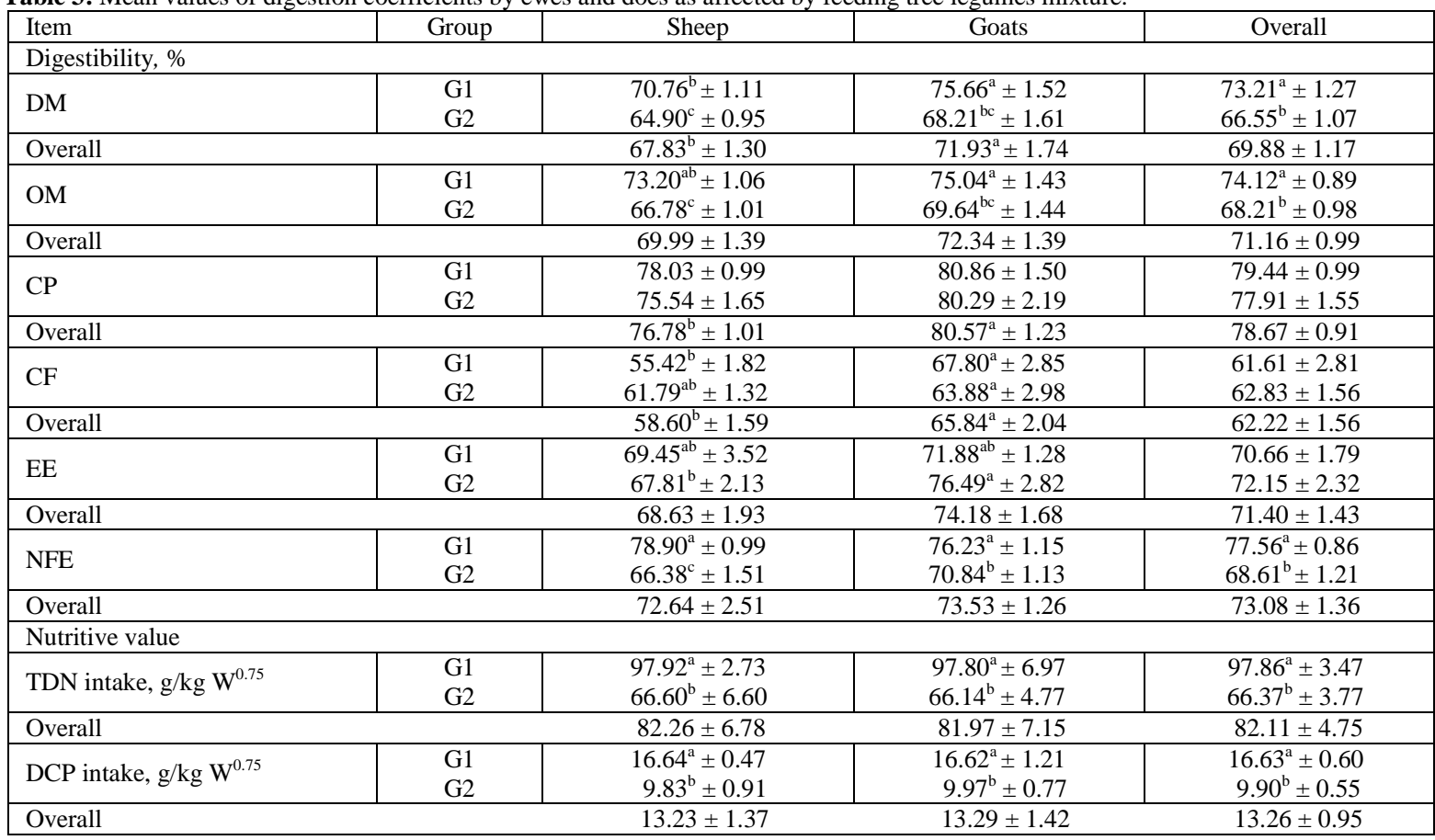

G1, animals fed on alfalfa + CFM (control group).

$\mathrm{G} 2$, animals fed on a mixture of $A$. saligna $(50 \%)+P$. juliflora $(25 \%)+$ L. leucocephala $(25 \%)+\mathrm{CFM}$.

$\mathrm{a}, \mathrm{b}, \mathrm{c}=$ values in the same column or row within a certain trait with different super scripts are significantly differed $(\mathrm{P}<0.05)$. 


\section{Nitrogen utilization:}

Nitrogen intake, excretion and balance $\left(\mathrm{g} / \mathrm{kg} \mathrm{W}^{0.75}\right)$ are presented in Table 4. Overall nitrogen intake was similar between both species and was higher $(\mathrm{P}<0.05)$ for animals fed G1 vs. those fed G2 $(2.66 \mathrm{vs} .1 .57 \mathrm{~g} / \mathrm{kg}$ $\mathrm{W}^{0.75}$ respectively). At similar trend, overall feacal, urinary and total nitrogen excretion $\left(\mathrm{g} / \mathrm{kg} \mathrm{W}{ }^{0.75}\right)$ were closed between both species and were higher $(\mathrm{P}<0.05)$ for animals fed G1 vs. those fed G2 (55 vs. 41, 1.03 vs. 34 and $1.58 \mathrm{vs} .0 .75 \mathrm{~g} / \mathrm{kg} \mathrm{W}^{0.75}$ respectively) due to the greater supply of soluble nitrogen in the rumen. Also, nitrogen balance was similar between both species and was insignificant higher for those fed G1 vs. G2. Results are in agreement with those reported by [52] who indicated that goats would be able to utilize nitrogen more efficiently than sheep. The decline in nitrogen retention of animals fed tree legumes mixture vs. alfalfa hay might be due to the lack of soluble nitrogen or low digestibility in the basal diets and high tannins concentrations in browse species have been associated with reduced nitrogen retention [53, 54]. Conversely, diets high in condensed tannins can also increase post-ruminal absorption of nitrogen [55], decrease in fecal nitrogen excretion [56] and increase $\mathrm{N}$ retention [57].

Table 4: Nitrogen utilization by ewes and does as affected by feeding tree legumes mixture.

\begin{tabular}{|c|c|c|c|c|}
\hline Item & Group & Sheep & Goats & Overall \\
\hline Nitrogen intake $\left(\mathrm{g} / \mathrm{kg} \mathrm{W} \mathrm{W}^{0.75}\right)$ & $\begin{array}{l}\text { G1 } \\
\text { G2 }\end{array}$ & $\begin{array}{l}2.66^{\mathrm{a}} \pm 0.07 \\
1.57^{\mathrm{b}} \pm 0.15\end{array}$ & $\begin{array}{l}2.66^{\mathrm{a}} \pm 0.19 \\
1.56^{\mathrm{b}} \pm 0.11\end{array}$ & $\begin{array}{l}2.66^{\mathrm{a}} \pm 0.10 \\
1.57^{\mathrm{b}} \pm 0.09\end{array}$ \\
\hline Overall & & $2.11 \pm 0.22$ & $2.11 \pm 0.23$ & $2.11 \pm 0.15$ \\
\hline Feacal N (g/kg W $\left.{ }^{0.75}\right)$ & $\begin{array}{l}\text { G1 } \\
\text { G2 }\end{array}$ & $\begin{array}{l}0.59^{\mathrm{a}} \pm 0.04 \\
0.49^{\mathrm{a}} \pm 0.06\end{array}$ & $\begin{array}{l}0.51^{\mathrm{a}} \pm 0.03 \\
0.33^{\mathrm{b}} \pm 0.06\end{array}$ & $\begin{array}{l}0.55^{\mathrm{a}} \pm 0.03 \\
0.41^{\mathrm{b}} \pm 0.05\end{array}$ \\
\hline Overall & & $0.54 \pm 0.04$ & $0.42 \pm 0.05$ & $0.48 \pm 0.03$ \\
\hline Urinary $\mathrm{N}\left(\mathrm{g} / \mathrm{kg} \mathrm{W}^{0.75}\right)$ & $\begin{array}{l}\text { G1 } \\
\text { G2 }\end{array}$ & $\begin{array}{l}1.02^{\mathrm{a}} \pm 0.12 \\
0.29^{\mathrm{b}} \pm 0.06\end{array}$ & $\begin{array}{l}1.05^{\mathrm{a}} \pm 0.18 \\
0.39^{\mathrm{b}} \pm 0.05\end{array}$ & $\begin{array}{l}1.03^{\mathrm{a}} \pm 0.10 \\
0.34^{\mathrm{b}} \pm 0.04\end{array}$ \\
\hline Overall & & $0.65 \pm 0.15$ & $0.72 \pm 0.15$ & $0.68 \pm 0.10$ \\
\hline Total $\mathrm{N}$ execration $\left(\mathrm{g} / \mathrm{kg} \mathrm{W}^{0.75}\right)$ & $\begin{array}{l}\text { G1 } \\
\text { G2 } \\
\end{array}$ & $\begin{array}{r}1.61^{\mathrm{a}} \pm 0.15 \\
0.78^{\mathrm{b}} \pm 0.09 \\
\end{array}$ & $\begin{array}{l}1.56^{\mathrm{a}} \pm 0.19 \\
0.72^{\mathrm{b}} \pm 0.11\end{array}$ & $\begin{array}{l}1.58^{\mathrm{a}} \pm 0.11 \\
0.75^{\mathrm{b}} \pm 0.07\end{array}$ \\
\hline Overall & & $1.19 \pm 0.17$ & $1.14 \pm 0.19$ & $1.16 \pm 0.12$ \\
\hline Nitrogen balance $\left(\mathrm{g} / \mathrm{kg} \mathrm{W}^{0.75}\right)$ & $\begin{array}{l}\text { G1 } \\
\text { G2 }\end{array}$ & $\begin{array}{l}1.05 \pm 0.11 \\
0.79 \pm 0.09\end{array}$ & $\begin{array}{l}1.11 \pm 0.23 \\
0.84 \pm 0.06\end{array}$ & $\begin{array}{l}1.08 \pm 0.12 \\
0.81 \pm 0.05\end{array}$ \\
\hline Overall & & $0.92 \pm 0.08$ & $0.97 \pm 0.12$ & $0.94 \pm 0.07$ \\
\hline
\end{tabular}

G1, animals fed on alfalfa + CFM (control group).

$\mathrm{G} 2$, animals fed on a mixture of $A$. saligna $(50 \%)+$ P. juliflora $(25 \%)+$ L. leucocephala $(25 \%)+$ CFM.

$\mathrm{a}, \mathrm{b}=$ values in the same column or row within a certain trait with different super scripts are significantly differed $(\mathrm{P}<0.05)$.

\section{Water utilization:}

Water utilization by sheep and goats where summarized in Table 5. Data indicated that goats had slightly higher in overall drinking, metabolic and total water intake $\left(\mathrm{ml} / \mathrm{kg} \mathrm{W}^{0.82}\right)$ compared to sheep $(107 \mathrm{vs} .97,28.24$ vs. 27.08 and 193 vs. $185 \mathrm{ml} / \mathrm{kg} \mathrm{W}^{0.82}$, respectively) while animals fed G1 had higher $(\mathrm{P}<0.05)$ in overall combined, metabolic and total water intake $\left(\mathrm{ml} / \mathrm{kg} \mathrm{W}^{0.82}\right)$ than those fed $\mathrm{G} 2$ (107.5 vs. 11.68, 34.09 vs. 21.23 and 229 vs. $149 \mathrm{ml} / \mathrm{kg} \mathrm{W}^{0.82}$, respectively). At similar trend, overall urinary, execration and water balance $\left(\mathrm{ml} / \mathrm{kg} \mathrm{W}^{0.82}\right)$ were similar between goats and sheep and were higher $(\mathrm{P}<0.05)$ for those fed alfalfa hay vs. tree legumes mixture (60.11 vs. $33.15,85.53$ vs. 58.21 and 143 vs. $91 \mathrm{ml} / \mathrm{kg} \mathrm{W}^{0.82}$, respectively). In corroboration with previous findings by [58] who reported that, sheep and goats consumed comparable amounts of water intake but goats was excreted more feacal and urinary water than sheep. Unlike wise, there are other reports noted that sheep was higher in water intake vs. goats $[59,60,61,62]$. 
Table 5: Water utilization by ewes and does fed diets containing tree legume mixture.

\begin{tabular}{|c|c|c|c|c|}
\hline Item & Group & Sheep & Goats & Overall \\
\hline Drinking water $\left(\mathrm{ml} / \mathrm{kg} \mathrm{W}^{0.82}\right)$ & $\begin{array}{l}\text { G1 } \\
\text { G2 }\end{array}$ & $\begin{array}{c}79.42^{\mathrm{b}} \pm 11.35 \\
115^{\mathrm{a}} \pm 6.26\end{array}$ & $\begin{array}{c}97.87^{\mathrm{ab}} \pm 11.95 \\
118^{\mathrm{a}} \pm 5.77\end{array}$ & $\begin{array}{r}88.64^{\mathrm{b}} \pm 8.39 \\
116^{\mathrm{a}} \pm 3.98\end{array}$ \\
\hline \multicolumn{2}{|l|}{ Overall } & $97 \pm 8.98$ & $107 \pm 7.20$ & $102 \pm 5.73$ \\
\hline Combined water $\left(\mathrm{ml} / \mathrm{kg} \mathrm{W}^{0.82}\right)$ & $\begin{array}{l}\text { G1 } \\
\text { G2 }\end{array}$ & $\begin{array}{r}107^{\mathrm{a}} \pm 3.53 \\
16.00^{\mathrm{b}} \pm 2.65\end{array}$ & $\begin{array}{l}108^{\mathrm{a}} \pm 10.25 \\
7.37^{\mathrm{b}} \pm 0.64\end{array}$ & $\begin{array}{l}107.5^{\mathrm{a}} \pm 5.02 \\
11.68^{\mathrm{b}} \pm 2.06\end{array}$ \\
\hline Overall & & $61.50 \pm 17.31$ & $57.68 \pm 19.59$ & $59.59 \pm 12.64$ \\
\hline *Metabolic water $\left(\mathrm{ml} / \mathrm{kg} \mathrm{W}^{0.82}\right)$ & $\begin{array}{l}\text { G1 } \\
\text { G2 }\end{array}$ & $\begin{array}{r}33.34^{\mathrm{a}} \pm 0.85 \\
20.82^{\mathrm{b}} \pm 1.85\end{array}$ & $\begin{array}{l}34.84^{\mathrm{a}} \pm 3.13 \\
21.64^{\mathrm{b}} \pm 1.30\end{array}$ & $\begin{array}{l}34.09^{\mathrm{a}} \pm 1.53 \\
21.23^{\mathrm{b}} \pm 1.06\end{array}$ \\
\hline Overall & & $27.08 \pm 2.55$ & $28.24 \pm 2.95$ & $27.66 \pm 1.89$ \\
\hline Total water intake $\left(\mathrm{ml} / \mathrm{kg} \mathrm{W}^{0.82}\right)$ & $\begin{array}{l}\text { G1 } \\
\text { G2 }\end{array}$ & $\begin{array}{l}219^{\mathrm{a}} \pm 8.89 \\
151^{\mathrm{b}} \pm 10.19\end{array}$ & $\begin{array}{l}240^{\mathrm{a}} \pm 29.04 \\
147^{\mathrm{b}} \pm 7.89\end{array}$ & $\begin{array}{l}229^{\mathrm{a}} \pm 14.19 \\
149^{\mathrm{b}} \pm 6.32\end{array}$ \\
\hline \multicolumn{2}{|l|}{ Overall } & $185 \pm 13.38$ & $193 \pm 21.08$ & $189 \pm 12.06$ \\
\hline Feacal water $\left(\mathrm{ml} / \mathrm{kg} \mathrm{W}^{0.82}\right)$ & $\begin{array}{l}\text { G1 } \\
\text { G2 }\end{array}$ & $\begin{array}{l}26.13 \pm 2.50 \\
23.75 \pm 3.33\end{array}$ & $\begin{array}{l}24.72 \pm 6.32 \\
26.38 \pm 4.14\end{array}$ & $\begin{array}{l}25.42 \pm 3.16 \\
25.06 \pm 2.51\end{array}$ \\
\hline \multicolumn{2}{|l|}{ Overall } & $24.94 \pm 1.98$ & $25.55 \pm 3.51$ & $25.24 \pm 1.95$ \\
\hline Urinary water $\left(\mathrm{ml} / \mathrm{kg} \mathrm{W}^{0.82}\right)$ & $\begin{array}{l}\text { G1 } \\
\text { G2 }\end{array}$ & $\begin{array}{l}61.83^{\mathrm{a}} \pm 4.93 \\
27.72^{\mathrm{b}} \pm 1.74\end{array}$ & $\begin{array}{c}58.39^{\mathrm{a}} \pm 8.53 \\
38.59^{\mathrm{ab}} \pm 12.50\end{array}$ & $\begin{array}{l}60.11^{\mathrm{a}} \pm 4.60 \\
33.15^{\mathrm{b}} \pm 6.19\end{array}$ \\
\hline \multicolumn{2}{|l|}{ Overall } & $44.77 \pm 6.89$ & $48.49 \pm 7.94$ & $46.63 \pm 5.10$ \\
\hline Total execration $\left(\mathrm{ml} / \mathrm{kg} \mathrm{W}^{0.82}\right)$ & $\begin{array}{l}\text { G1 } \\
\text { G2 }\end{array}$ & $\begin{array}{l}87.96 \pm 5.28 \\
51.47 \pm 4.47\end{array}$ & $\begin{array}{l}83.11 \pm 14.49 \\
64.97 \pm 15.73\end{array}$ & $\begin{array}{r}85.53^{\mathrm{a}} \pm 7.20 \\
58.21^{\mathrm{b}} \pm 7.99\end{array}$ \\
\hline \multicolumn{2}{|l|}{ Overall } & $69.71 \pm 7.60$ & $74.04 \pm 10.48$ & $71.87 \pm 6.28$ \\
\hline Water balance $\left(\mathrm{ml} / \mathrm{kg} \mathrm{W}^{0.82}\right)$ & $\begin{array}{l}\text { G1 } \\
\text { G2 }\end{array}$ & $\begin{array}{l}131^{\mathrm{ab}} \pm 4.58 \\
100^{\mathrm{bc}} \pm 7.32\end{array}$ & $\begin{array}{r}156^{\mathrm{a}} \pm 16.59 \\
82.03^{\mathrm{c}} \pm 11.30\end{array}$ & $\begin{array}{r}143^{\mathrm{a}} \pm 8.46 \\
91^{\mathrm{b}} \pm 7.76\end{array}$ \\
\hline Overall & & $115 \pm 6.34$ & $119 \pm 15.50$ & $117 \pm 8.11$ \\
\hline
\end{tabular}

G1, animals fed on alfalfa + CFM (control group).

G2, animals fed on a mixture of $A$. saligna $(50 \%)+P$. juliflora $(25 \%)+$ L. leucocephala $(25 \%)+\mathrm{CFM}$.

*Metabolic water was calculated from TDN intake a yield of $0.6 \mathrm{~g}$. water per g. [63].

$\mathrm{a}, \mathrm{b}, \mathrm{c}=$ values in the same column or row within a certain trait with different super scripts are significantly differed $(\mathrm{P}<0.05)$.

\section{Ruminal parameters:}

Ammonia (NH3-N mg/100 ml) and volatile fatty acids (VFA's m. equiv. $/ 100 \mathrm{ml}$ ) concentrations are presented in Table 6. Goats had higher $(\mathrm{P}<0.05)$ overall ammonia NH3-N than sheep (35.43 vs. $27.90 \mathrm{mg} / 100$ $\mathrm{ml}$, respectively). In agreement with [64] who noted that, goats where considered to have a higher concentration of rumen ammonia, which could lead to an improved fiber digestion. However, animals fed G1 showed higher $(\mathrm{P}<0.05)$ NH3-N than those fed G2 (35.26 vs. $28.07 \mathrm{mg} / 100 \mathrm{ml}$ respectively). Lower concentrations of NH3-N in rumen fluid found with Acacia spp and Leucaena spp could be attributed to the inhibition of rumen protein degradation and deamination processes by condensed tannins $[65,66,67]$. Sheep had higher $(\mathrm{P}<0.05)$ overall ruminal TVFA's than goats (11.18 vs.7.46 m. equiv. /100 ml respectively) and was lower $(\mathrm{P}<0.05)$ for animals fed G2 vs. G1 (7.27 vs. $11.37 \mathrm{~m}$. equiv. /100 ml respectively). In corporation with previous findings by [68] who noted that sheep had greater $(\mathrm{P}<0.05)$ in mean ruminal VFA concentrations compared with goats for all diets. The low concentrations of ruminal TVFA's in rumen could be attributed to the reduce energy supply to the host [69].

Table 6: Rumen ammonia $\left(\mathrm{NH}_{3}-\mathrm{N}, \mathrm{mg} / 100 \mathrm{ml}\right)$ and total volatile fatty acids (VFA's, m. equiv. /100 ml) concentration of ewes and does as affected by feeding tree legumes mixture.

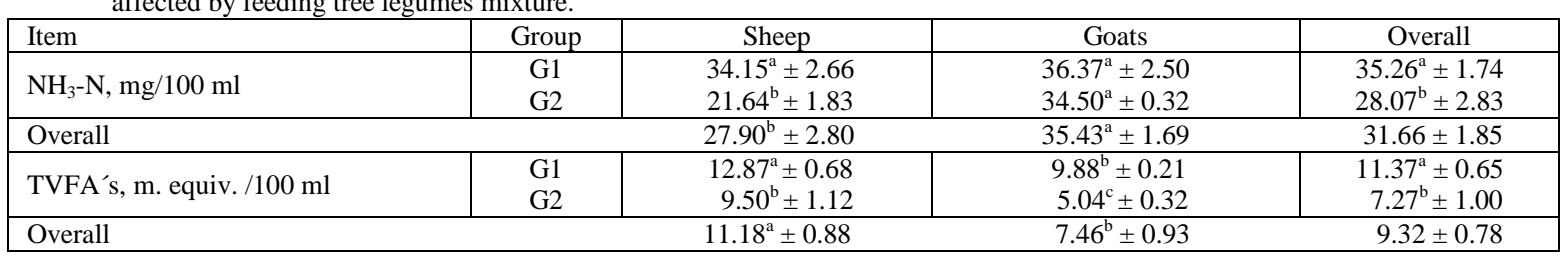

G1, animals fed on alfalfa + CFM (control group).

$\mathrm{G} 2$, animals fed on a mixture of A. saligna $(50 \%)+$ P. juliflora $(25 \%)+$ L. leucocephala $(25 \%)+$ CFM.

$\mathrm{a}, \mathrm{b}, \mathrm{c}=$ values in the same column or row within a certain trait with different super scripts are significantly differed $(\mathrm{P}<0.05)$.

Live body weight changes:

Body weight changes $(\mathrm{kg})$ of ewes and does are presented in Table 7. The obtained results showed that values of body weight change $(\mathrm{kg})$ and relative body weight $(\%)$ at end pregnancy period were insignificant between both species and feeding treatments. This agreement with finding of [70] they reported that, final body weight where similar in goats fed a diet having $50 \%$ of dietary Acacia karroo compared with other treatment groups. Moreover, [71] indicated that Awassi ewes fed Sesbania aculeate had a comparable live body weight of ewes for control group. In similar, [72] found that goats fed Leucaena leucocephala fodder for three months, maintained their body weights throughout the experimental period without any where deleterious effect. Similar results were reported by [73] were feeding pelleted diets containing different proportions of sun-dried $L$. 
leucocephala led to non-significant differences final body weight and average body weight changes of Jamunapari male goats.

Table 7: Body weight changes of pregnant ewes and does as affected by feeding tree legume mixture.

\begin{tabular}{|c|c|c|c|c|}
\hline Item & Group & Sheep & Goats & Overall \\
\hline N0. of animals & & 18 & 18 & \\
\hline \multirow{2}{*}{ Initial body weight (kg) } & G1 & $26.42 \pm 1.17$ & $25.93 \pm 1.50$ & $26.17 \pm 0.92$ \\
\hline & $\mathrm{G} 2$ & $26.71 \pm 0.46$ & $25.00 \pm 2.41$ & $25.85 \pm 1.20$ \\
\hline Overall & & $26.56 \pm 0.61$ & $25.46 \pm 1.40$ & $26.01 \pm 0.74$ \\
\hline \multirow{2}{*}{ End of pregnancy $(\mathrm{kg})$} & G1 & $32.97 \pm 1.66$ & $30.86 \pm 1.62$ & $31.91 \pm 1.15$ \\
\hline & G2 & $33.29 \pm 0.96$ & $30.71 \pm 2.40$ & $32.00 \pm 1.29$ \\
\hline Overall & & $33.13 \pm 0.92$ & $30.78 \pm 1.39$ & $31.95 \pm 0.85$ \\
\hline \multirow{2}{*}{ Body weight change (kg) } & G1 & $6.55 \pm 0.80$ & $4.93 \pm 0.58$ & $5.74 \pm 0.52$ \\
\hline & $\mathrm{G} 2$ & $6.58 \pm 0.98$ & $5.71 \pm 0.58$ & $6.14 \pm 0.56$ \\
\hline Overall & & $6.56 \pm 0.61$ & $5.32 \pm 0.41$ & $5.94 \pm 0.38$ \\
\hline \multirow{2}{*}{ "Relative Body weight (\%) } & G1 & $24.79 \pm 2.68$ & $19.01 \pm 2.32$ & $21.90 \pm 1.86$ \\
\hline & $\mathrm{G} 2$ & $24.63 \pm 3.84$ & $22.84 \pm 3.77$ & $23.73 \pm 2.59$ \\
\hline Overall & & $24.71 \pm 2.25$ & $20.92 \pm 2.24$ & $23.81 \pm 1.58$ \\
\hline
\end{tabular}

G1, animals fed on alfalfa + CFM (control group).

$\mathrm{G} 2$, animals fed on a mixture of acacia $(50 \%)+$ prosopis $(25 \%)+$ leucaena $(25 \%)+\mathrm{CFM} ;{ }^{*}$ Relative body weight $=$ Body weight change $(\mathrm{kg}) /$ Initial body weight $(\mathrm{kg}) \times 100$.

$\mathrm{a}, \mathrm{b}=$ Values in the same column or row within a certain trait with different super scripts are not significantly differed $(\mathrm{P}<0.05)$.

Price cost of feed intake:

Cost of feed intake (h/d, L.E.) of ewes and does are reported in Table 8. Data showed that the lower at feed cost was noticed for animals fed G2 than those fed G1 in daily total feed cost, feed cost of kg total dry matter intake (TDMI) and TDN. Similarly, feed cost of $\mathrm{Kg} \mathrm{W}^{0.75}$ (L.E.) and relative cost of $\mathrm{kg}$ DMI (\%) were lower price of mixture vs. control and was similar between both species. Our tree legumes mixture recorded a reductions in feed cost of $\mathrm{Kg} \mathrm{W}^{0.75}$ about $53 \%$ for sheep and $54 \%$ for goats compared with control group due to the low price of tree legumes mixture compared with alfalfa. The results are in agreement with those reported by $[74,75,76]$ they found that the feed cost was relatively lower than the control when sheep and goats fed rations contained $30-40 \%$ acacia.

Table 8: Price of feed intake by ewes and does during early pregnancy as affected by feeding salt tolerant plants

\begin{tabular}{|c|c|c|c|}
\hline Item & Group & Sheep & Goats \\
\hline \multicolumn{4}{|l|}{ Price of feed intake, h/d, L.E. } \\
\hline Total feed cost & $\begin{array}{l}\mathrm{G} 1 \\
\mathrm{G} 2\end{array}$ & $\begin{array}{l}6.00 \\
2.57\end{array}$ & $\begin{array}{l}4.97 \\
2.35\end{array}$ \\
\hline Feed cost of Kg DMI (L.E.) & $\begin{array}{l}\text { G1 } \\
\text { G2 }\end{array}$ & $\begin{array}{l}3.92 \\
2.81\end{array}$ & $\begin{array}{l}3.98 \\
2.91\end{array}$ \\
\hline Feed cost of $\mathrm{Kg}$ TDN (L.E.) & $\begin{array}{l}\text { G1 } \\
\text { G2 }\end{array}$ & $\begin{array}{l}5.89 \\
5.31\end{array}$ & $\begin{array}{l}5.72 \\
5.08\end{array}$ \\
\hline Feed cost of $\mathrm{Kg} \mathrm{W}^{0.75}$ (L.E.) & $\begin{array}{l}\text { G1 } \\
\text { G2 }\end{array}$ & $\begin{array}{l}0.40 \\
0.19\end{array}$ & $\begin{array}{l}0.39 \\
0.18\end{array}$ \\
\hline Relative feed cost of $\mathrm{Kg}$ DMI (\%) & $\begin{array}{l}\text { G1 } \\
\text { G2 }\end{array}$ & $\begin{array}{c}100 \\
72\end{array}$ & $\begin{array}{c}100 \\
73\end{array}$ \\
\hline
\end{tabular}

The price of concentrate feed mixture, alfalfa and mixture of Acacia saligna (50\%) + Prosopis juliflora (25\%) + Leucaena leucocephala $(25 \%)=4800,3500$ and 800 L.E /ton, respectively.

\section{Blood parameters:}

Data of blood parameters showed in Table 9. Sheep had slightly insignificant lower concentrations of overall total protein and globulin than goats (6.50 vs. 6.96 and 3.38 vs. 3.97 (g/dl), respectively) while it showed insignificant higher of overall albumin and albumin/ globulin ration (A/g ratio) than goats (3.12 vs. 2.99 and 0.94 vs. 0.75 , respectively) with no significant different among treatments. These findings were in harmony with those reported by $[77,78,79,80]$. Also sheep had slightly insignificant higher concentrations of overall urea and creatinine than goats (41.24 vs. 39.87 and 1.25 vs. $1.19(\mathrm{mg} / \mathrm{dl})$, respectively) with insignificant effect between experimental diets. In similar, [80] found that animals fed mixture of sun-dried acacia, prosopis and leucaena showed lower mean concentration of urea and creatinine compared with control group. It might be due to the high presence of condensed tannins, which led to reduce the ruminal proteins degradation in turn and caused a reduction of urea nitrogen levels in sheep and goats [81]. At the same trend, goats recorded insignificant higher values of overall AST and ALT compared to sheep (24.06 vs. 19.32 and 29.31 vs. 23.49 (IU/l), respectively). The results were in agreement with [80]. In general, the increase of ALT or AST activity might be cause by high tannins [82]. Also goats had higher overall total lipids and glucose than sheep (2.94 vs. 2.75 (g/l) and 44.45 vs. 39.65 (mg/l), respectively) but sheep showed higher overall cholesterol than goats (1.25 vs. 1.19 (mg/dl), respectively) with no differences were observed between treatments. In agreement with [80] who demonstrated that feeding tree legumes mixture decreased mean values of plasma total lipids, cholesterol and glucose 
compared with alfalfa hay. The reduction in total lipids might be attributed that such tree legumes mixture and low fat contents in such desert plants. Moreover, this decrease in blood cholesterol and glucose levels might be attributed to the anti-nutritional factors in these plants $[83,84]$.

Table 9: Mean values of some blood metabolites concentration of ewes and does as affected by feeding tree legumes mixture.

\begin{tabular}{|c|c|c|c|c|}
\hline Item & Group & Sheep & Goats & Overall \\
\hline Total proteins (g/dl) & $\begin{array}{l}\text { G1 } \\
\text { G2 }\end{array}$ & $\begin{array}{l}7.42 \pm 1.32 \\
5.59 \pm 0.63\end{array}$ & $\begin{array}{l}7.19 \pm 0.94 \\
6.72 \pm 0.79\end{array}$ & $\begin{array}{l}7.31 \pm 0.73 \\
6.16 \pm 0.52\end{array}$ \\
\hline Overall & & $6.50 \pm 0.77$ & $6.96 \pm 0.56$ & $6.73 \pm 0.46$ \\
\hline Albumin (g/dl) & $\begin{array}{l}\text { G1 } \\
\text { G2 } \\
\end{array}$ & $\begin{array}{l}3.36 \pm 0.81 \\
2.88 \pm 0.43\end{array}$ & $\begin{array}{l}3.33 \pm 0.32 \\
2.64 \pm 0.61\end{array}$ & $\begin{array}{l}3.35 \pm 0.39 \\
2.76 \pm 0.34\end{array}$ \\
\hline Overall & & $3.12 \pm 0.42$ & $2.99 \pm 0.35$ & $3.06 \pm 0.26$ \\
\hline Globulin (g/dl) & $\begin{array}{l}\text { G1 } \\
\text { G2 }\end{array}$ & $\begin{array}{l}4.06 \pm 0.75 \\
2.71 \pm 0.89 \\
\end{array}$ & $\begin{array}{l}3.86 \pm 1.26 \\
4.08 \pm 0.38 \\
\end{array}$ & $\begin{array}{l}3.96 \pm 0.65 \\
3.40 \pm 0.53\end{array}$ \\
\hline Overall & & $3.38 \pm 0.60$ & $3.97 \pm 0.59$ & $3.68 \pm 0.41$ \\
\hline $\mathrm{A} / \mathrm{G}$ ratio & $\begin{array}{l}\text { G1 } \\
\text { G2 }\end{array}$ & $\begin{array}{l}0.82 \pm 0.23 \\
1.06 \pm 1.06\end{array}$ & $\begin{array}{l}0.86 \pm 0.36 \\
0.65 \pm 0.14\end{array}$ & $\begin{array}{l}0.84 \pm 0.20 \\
0.81 \pm 0.54\end{array}$ \\
\hline Overall & & $0.94 \pm 0.52$ & $0.75 \pm 0.20$ & $0.82 \pm 0.27$ \\
\hline Urea (mg/dl) & $\begin{array}{l}\text { G1 } \\
\text { G2 } \\
\end{array}$ & $\begin{array}{l}41.03 \pm 6.28 \\
41.45 \pm 4.94 \\
\end{array}$ & $\begin{array}{l}46.25 \pm 3.52 \\
33.48 \pm 3.99\end{array}$ & $\begin{array}{l}43.64 \pm 3.42 \\
37.47 \pm 3.35 \\
\end{array}$ \\
\hline Overall & & $41.24 \pm 3.58$ & $39.87 \pm 3.71$ & $40.55 \pm 2.47$ \\
\hline Creatinine (mg/dl) & $\begin{array}{l}\text { G1 } \\
\text { G2 }\end{array}$ & $\begin{array}{l}1.31 \pm 0.13 \\
1.19 \pm 0.11\end{array}$ & $\begin{array}{l}1.29 \pm 0.02 \\
1.08 \pm 0.03\end{array}$ & $\begin{array}{l}1.30 \pm 0.06 \\
1.13 \pm 0.06\end{array}$ \\
\hline Overall & & $1.25 \pm 0.08$ & $1.19 \pm 0.05$ & $1.22 \pm 0.05$ \\
\hline AST (IU/l) & $\begin{array}{l}\text { G1 } \\
\text { G2 } \\
\end{array}$ & $\begin{array}{l}18.48 \pm 5.35 \\
20.16 \pm 6.05 \\
\end{array}$ & $\begin{array}{l}20.28 \pm 2.99 \\
27.84 \pm 1.18 \\
\end{array}$ & $\begin{array}{l}19.38 \pm 2.77 \\
24.00 \pm 3.25 \\
\end{array}$ \\
\hline Overall & & $19.32 \pm 3.63$ & $24.06 \pm 2.22$ & $21.69 \pm 2.15$ \\
\hline ALT (IU/l) & $\begin{array}{l}\text { G1 } \\
\text { G2 }\end{array}$ & $\begin{array}{l}23.40 \pm 5.49 \\
23.58 \pm 1.35\end{array}$ & $\begin{array}{l}26.47 \pm 0.32 \\
32.14 \pm 4.60\end{array}$ & $\begin{array}{l}24.94 \pm 2.55 \\
27.86 \pm 2.87\end{array}$ \\
\hline Overall & & $23.49 \pm 2.53$ & $29.31 \pm 2.42$ & $26.40 \pm 1.88$ \\
\hline ALP (IU/l) & $\begin{array}{l}\text { G1 } \\
\text { G2 } \\
\end{array}$ & $\begin{array}{l}133.71 \pm 6.17 \\
133.74 \pm 3.87\end{array}$ & $\begin{array}{l}134.26 \pm 3.39 \\
129.39 \pm 4.28\end{array}$ & $\begin{array}{l}133.99 \pm 3.15 \\
131.56 \pm 2.76\end{array}$ \\
\hline Overall & & $133.72 \pm 3.26$ & $131.83 \pm 2.67$ & $132.78 \pm 2.03$ \\
\hline Total lipids (g/l) & $\begin{array}{l}\text { G1 } \\
\text { G2 }\end{array}$ & $\begin{array}{r}3.03^{\mathrm{ab}} \pm 0.30 \\
2.47^{\mathrm{b}} \pm 0.06 \\
\end{array}$ & $\begin{array}{r}3.20^{\mathrm{a}} \pm 0.07 \\
2.67^{\mathrm{ab}} \pm 0.19\end{array}$ & $\begin{array}{l}3.11^{\mathrm{a}} \pm 0.14 \\
2.57^{\mathrm{b}} \pm 0.10\end{array}$ \\
\hline Overall & & $2.75 \pm 0.18$ & $2.94 \pm 0.15$ & $2.84 \pm 0.11$ \\
\hline Cholesterol (mg/dl) & $\begin{array}{l}\text { G1 } \\
\text { G2 }\end{array}$ & $\begin{array}{l}99.63 \pm 0.87 \\
99.09 \pm 5.85\end{array}$ & $\begin{array}{l}99.00 \pm 4.59 \\
90.04 \pm 2.91\end{array}$ & $\begin{array}{l}99.32 \pm 2.09 \\
94.57 \pm 3.55\end{array}$ \\
\hline Overall & & $99.36 \pm 2.65$ & $94.52 \pm 3.15$ & $96.94 \pm 2.09$ \\
\hline Glucose (mg/l) & $\begin{array}{l}\text { G1 } \\
\text { G2 } \\
\end{array}$ & $\begin{array}{l}44.26 \pm 5.19 \\
35.04 \pm 2.89 \\
\end{array}$ & $\begin{array}{l}48.55 \pm 2.54 \\
40.34 \pm 6.09\end{array}$ & $\begin{array}{l}46.41 \pm 2.76 \\
37.69 \pm 3.24 \\
\end{array}$ \\
\hline Overall & & $39.65 \pm 3.36$ & $44.45 \pm 3.48$ & $42.05 \pm 2.42$ \\
\hline
\end{tabular}

G1, animals fed on alfalfa + CFM (control group).

$\mathrm{G} 2$, animals fed on a mixture of $A$. saligna $(50 \%)+$ P. juliflora $(25 \%)+$ L. leucocephala $(25 \%)+$ CFM.

$\mathrm{a}, \mathrm{b}=$ values in the same column or row within a certain trait with different super scripts are significantly differed $(\mathrm{P}<0.05)$.

\section{Conclusion:}

In brief, we can say that using sun-dried chopped mixture contained 50\% Acacia saligna, 25\% Prosopis juliflora and $25 \%$ Leucaena leucocephala as non-traditional could to uses a solve of some problems and providing as good alternative feed resources mainly in summer and autumn seasons when the other conventional forage resources are shortage without having any adverse effect on nutrient intake and performance of Barki sheep and doe Shami goats under desert conditions of South Sinai, Egypt.

\section{ACKNOWLEDGMENT}

This experiment is a part of the regional project titled "Adaptation to climate changes in WANA marginal environments through sustainable crop and livestock diversification" which is funded by International Center for Biosaline Agricultural (ICBA), UAE.

\section{REFERENCES}

[1] El Shaer, H.M., 2015. Adaptation to climate change in decertified lands of the marginal regions in Egypt through sustainable crop and livestock diversification systems. Sciences in Cold and Arid Regions, 7 (1): 16-22.

[2] Makkar, H.P.S., 2003. Effects and fate of tannins in ruminant animals, adaptation to tannins, and strategies to overcome detrimental effects of feeding tannin-rich feeds. Small Ruminant Research, (49): 241-256. 
[3] El-Shaer, H.M., 2010. Halophytes and salt-tolerant plants as potential forage for ruminants in the Near East region. Small Ruminant Research, 91: 3-12.

[4] Ben Salem, H., H. Nefzaoi, L. Ben Salem and J. Tisserand, 1999. Intake, digestibility, urinary excretion of purine derivatives and growth by sheep given fresh, air-dried or polyethlene glycolsoaked foliage of acacia cynophylla Land. J. Anim. Feed Sci. Technol., pp: 297-311.

[5] Safinaz, M.S., M.H. Ahmed and M.A. Ibrahim, 2010. Impact of feeding atriplex halimus and acacia saligna with different sources of energy on lambs performan. Egyptian J. Sheep Goat Sci., 5(1): 191-208.

[6] Moujahed, N., C. Kayoulib, A. Thewis, Y. Beckers and S. Rezgui, 2000. Effect of multinutrient blocks and polythylene glycol 4000 supplies on intake and digestion by sheep fed Acacia cyanophylla lindl. foliage based diets. J. Anim. Feed Sci. Technol., pp: 219-238.

[7] Moujahed, N., H. Ben Salem and C. Kayouli, 2005. Effects of frequency of polyethylene glycol and protein supplementation on intake and digestion of Acacia cyanophylla Lindl. foliage fed to sheep and goats. Small Rumin. Res., 56: 65-73.

[8] Shumuye, B. and T. Yayneshet, 2011. Effect of feeding treated Acacia Saligna (Labill.) H.L. Wendl. leaves on growth performance and digestibility in goats. J. Dryland Agric. 4(2): 341-347.

[9] Abdulrazak, S.A., T. Fujihara, J.K. Ondiek and E.R. Orskov, 2000. Nutritive evaluation of some Acacia tree leaves from Kenya. Animal Feed Science and Technology, 85: 89-98.

[10] Chaturvedi, O.H. and A. Sahoo, 2013. Nutrient utilization and rumen metabolism in sheep fed Prosopis juliflora pods and Cenchrus grass. Springer Plus, 2: 598.

[11] Sawal, R.K., R. Ratan and S.B.S. Yadav, 2004. Mesquite (Prosopis juliflora) Pods as a Feed Resource for Livestock- A Review. Asian Australas J Anim Sci., 17: 719-25.

[12] Pandya, P.R., G.R. Patel, R.S. Gupta, D.C. Patel, M.B. Pande and M.C. Desai, 2005. Effect of Wheat straw based total mixed ration with Prosopis juliflora pods (Mesquite pods) on performance of lactating cows. Inte J Cow Sci., 1: 66-72.

[13] Sharma, V., G.R. Purohit, R.S. Arya and M. Harsh, 2006. Evaluation of some complete rations in sheep incorporating unconventional feed resources of arid zone of India. Anim Nutr Feed Technol., 6: 135-141.

[14] Talpada, P.M., P.R. Pandya, G.R. Patel, D.C. Patel and M.C. Desai, 2002. Utilization of complete feds using Prosopis juliflora pods as a ration of growing crossbred calves. Indian J. Anim. Nutr. 19: 1-6.

[15] Aregheore, E.M., 1999. Nutritive and anti-nutritive value of some tree legumes used in ruminant livestock nutrition in Pacific Island countries. J South Pacific Agric, 6: 50-61.

[16] Barros-Rodríguez, M., J. Solorio-Sánchez and C. Sandoval-Castro, 2013. Effects of two intake levels of Leucaena leucocephala on rumen function of sheep. Trop Grassl Forrajes Tropicales, 1: 55-7.

[17] Nguyen, T.T.G., M. Wanapat, K. Phesatcha and S. Kang, 2017. Effect of inclusion of different levels of Leucaena silage on rumen microbial population and microbial protein synthesis in dairy steers fed on rice straw Asian-Australas J Anim Sci., pp: 1-6.

[18] Anbarasu, C., N. Dutta and K. Sharma, 2001. Use of leaf meal mixture as a protein supplement in the ration of goats fed wheat straw. Anim. Nutr. Feed Technol., 1: 113-123.

[19] Patra, A.K., K. Sharma, N. Dutta and A.K. Pattanaik, 2002. Effect of partial replacement of dietary protein by a leaf meal mixture containing Leucaena leucocephala, Morus alba and Azadirachta indica on performance of goats. Asian-Aust. J. Anim. Sci., 5 (12): 1732-1737.

[20] Kearl, I.C., 1982. Nutrients requirements in developing countries. Utah Agric. Exp. Stat., Utah State University, Logan, USA.

[21]AOAC., 2000. Official methods of analysis. 17th ed. Association of Official nalytical Chemists, Washington, DC, USA.

[22] Warner, A.C.J., 1964. Production of volatile fatty acids in the rumen methods of measurements Nutr. Abst. and Rev., 34: 339.

[23] Gornal, A.C., C.J. Bardawill and M.M. David, 1949. Kit Protein Egyptian American Co. for Laboratory Services. J. Biol. Chem., 177: 751-755.

[24] Doumas, B.T., W.A. Watson and H.G. Biggs, 1971. Albumin standards and the measurement of serum albumin with bromcresol green. Clinca Chemica Acta, 31: 87- 96.

[25] Schmit, J.M., 1964. Thesis, Lyon.

[26] Roeschlau, P., E. Bernt and W. Gurber, 1974. Enzymatic determination of total cholesterol in serum. Zklin. Chem. Klin. Biochem., 12(5): 226.

[27] Tietz, N.W., 1986. Textbook of Clinical Chemistry. W. B. Saunders Co. London, Philadelphia, pp: 796.

[28] Reitman, S.M.D. and S. Frankel, 1957. A colorimeter method for determination of serum glutamic oxaloacetic acid and glutamic pyruvic acid transferees. Am. J. Clin. Path., 28: 56-63.

[29] Belfield, A. and D.M. Goldberg, 1971. Revised assay for serum phenyl phosphayatase activity using 4amino- antipyrine. Enzyme., 12: 561- 573.

[30] Fawcett, J.K. and J.E. Soctt, 1960. A rapid precise method for the determination of urea. J. Cline., Path., 13: 156. 
[31] Schirmeister, J., H. Willmann, H. Kiefer and W. Hallauer, 1964. Fuer und wider die brauchbarkeit der endogenen hrea tininclearance der fanktionellen nierendiagnostik. Dtsch. Med. Woschr, 89 (9): 1640-1647.

[32] SAS Institute, 2004. Statistical Analysis System, STAT/ user's guide, Release 9.1, SAS Institute, Cary NC. USA.

[33] Duncan, D.B., 1955. Multiple range and multiple F- test. Biometris, 11: 1-42.

[34] Salem, A., M. Salem, M. El-Adawya and P. Robinson, 2006. Nutritive evaluations of some browse tree foliages during the dry season: Secondary compounds, feed intake and in vivo digestibility in sheep and goats. Anim. Feed Sci. Technol., pp: 251-267.

[35] Food Agriculture Organization (FAO), 1992. Legume trees and other fodder trees as protein sources for livestock In: Speedy, A., Pugliese, P.L. (Eds.), Proceedingsd of the FAO Expert Consultation.

[36] Topps, J.H., 1992. Potential, composition and use of legume shrubs and trees as fodder for livestock in the tropics. J. Agric. Sci. Camb., 118: 1-8.

[37] Ben Salem, H., A. Nefzaoui, H. Makkarb, H. Hochlef, I. Ben Salem and L. Ben Salem, 2005a. Effect of early experience and adaptation period on voluntary intake, digestion, and growth in Barbarine lambs given tannin-containing (Acacia cyanophylla Lindl. Foliage) or tannin-free (Oaten hay) diets. J. Animal Feed Sci. Technol., pp: 59-77.

[38] Abdel-Fattah, Z.S., 2005. Impact of season of harvest on in vitro gas production and dry matter degradability of Acacia saligna leaves with inoculum from three ruminant species. Anim. Feed Sci. Technol., pp: 67-79.

[39]El Hag, M.G. and K.M. Al Shargi, 1998. Comparative Performance of Goats and Sheep Fed on High-fiber Pelleted Diets Supplemented with Different Nitrogen Sources. J. Appl. h i m. Res., 13: 179-184.

[40] Nefzaoui, A., H. Ben Salem, H. Abdouli and H. Ferchichi, 1993. Palatability of some Mediterranean shrubs. Comparison between browsing time and bacteria technique. In: FAO/CIHEAMWorkshop on Sheep and Goats Nutrition, Thessaloniki, Greece, pp: 99-109.

[41] Bartolom'e, J., J. Franch, J. Plaixats and N.G. Seligman, 1998. Diet selection by sheep and goats on Mediterranean heath woodland range. J. Range Manage. 51: 383-391.

[42] Rogosic, J., J.A. Pfister, F.D. Provenza and D. Grbesa, 2006. Sheep and goat preference for and Rome, Italy, Pp: 32-93.

[43] Ben Salem, H., I. Ben Salem and M.S. Ben Said, 2005. Effect of the level and frequency of PEG supply on intake, digestion, biochemical and clinical parameters by goats given kermes oak (Quercus coccifera L.)based diets. Small Rumin. Res., 56: 127-137.

[44] Ben Salem, H. and T. Smith, 2008. Feeding strategies to increase small ruminant production in dry environments. Small Ruminant Res., 77: 174-194.

[45] Agrawal1, A.R., S.A. Karim, A. Rajiv Kumar, Sahoo and P.J. John, 2014. Sheep and goat production: basic differences impact on climate and molecular tools for rumen microbiome study. Int. J. Curr. Microbiol. App. Sci., 3 (1): 684-706.

[46] Silanikove, N., N. Gilboa, I. Nir, A. Perevolotsky and Z. Nitsan, 1996. Effect of a daily supplementation of polyethylene glycol on intake and digestion of tannin-containing leaves (Quercus calliprinos, Pisticia lentiscus and Ceratonia siliqua) by goats. J. Agric. Food Chem., 44: 199-205.

[47] Santra, A., S.A. Karim, A.S. Mishra, O.H. Chaturvedi and R. Prasad, 1998. Rumen ciliate protozoa and fiber utilization in sheep and goats. Small Rumin. Res., 30: 13-18.

[48] Min, B.R., T.N. Barry, G.T. Attwood and W.C. Mcnabb, 2003. The effect of condensed tannins on the nutritive and health of ruminants fed fresh temperate forages: a review. Animal Feed Science and Technology, 106 (1-4): P. 3-19.

[49] Mlambo, V., U. Marume and C.S. Gajana, 2015. Utility of the browser's behavioural and physiological strategies in coping with dietary tannins: Are exogenous tannin inactivating treatments necessary. South African Journal of Animal Science., 45 (5): 441-451.

[50] Ortiz, L.T., C. Centeno and J. Tervino, 1993. Tannins in faba bean seeds: effect on the digestion of protein and amino acids in growing chicks. Animal Feed Science and Technology, 41: $271-278$.

[51] Streeter, M.N., G.M. Hill, D.G. Wagner, F.N. Owens and C.A. Hibberd, 1993. Effect of bird resistant and non bird resistant sorghum gain on amino acid digestion by beef heifers. J. Anim. Sci., 71: 1648- 1656.

[52] El-Meccawi, S., M. Kam, A. Brosh and A.A. Degen, 2008. Heat production and energy balance of sheep and goats fed sole diets of Acacia saligna and Medicago sativa. Small Rumin. Res., 75: 199-203.

[53] Reed, J.D., H. Soller and A. Woodward, 1990. Fodder tree and straw diets for sheep: intake, growth, digestibility and the effects of phenolics on nitrogen utilisation. Anim. Feed Sci. Technol., 30 (1-2): 39-50.

[54] Ben Salem, H., A. Nefzaoui, L. Ben Salem and J.L. Tisserand, 1997. Intake, digestibility in sheep given fresh or air-dried Acacia cynophylla Lindl. foliage. Ann. Zootech, 46: 361-374.

[55] Egea, A.V., L.I. Allegretti, S.A. Paez Lama, D.J. Grilli, M. Fucili, J.C. Guevara and J.J. Villalba, 2016. Diet mixing and condensed tannins help explain foraging preferen cesby Creole goats facing the physical and 
chemical diversity of native woody plants in the central Monte desert (Argentina). Animal Feed Science and Technology, 215: 47-57.

[56] Ndemanisho, E.E., L.A. Mtenga, E.F.C. Kimbi, A.E. Kimambo and E.J. Mtengati, 1998. Substitution of dry Leucaena leucocephala (DLL) for cotton seed cake (CSC) as a protein supplement to urea treated maize stover fed to dairy weaner goats. Anim. Feed Sci. Technol., 73: 365-374.

[57] Foroughbakhch, R., J.L. Hernández-Piñero, A. Carrillo-Parra, and A. Rocha-Estrada, 2013. Composition and animal preference for plants used for goat feeding in semiarid northeastern México. Journal of Animal and Plant Sciences, 23 (4): 1034-1040.

[58] Gihad, E.A., T.T.L. El gallad, A.E. Sooud, H.M. Abou El-Nasr and M.F.A. Farid, 1989. Feed and water intake, digestibility and nitrogen utilization by camels compared to sheep and goats fed low protein desert by-products Ciheam-Options Mediterraneennes Ser. Seminaries, 2: 75-81.

[59] Gihad, E.A., 1976. Intake, digestibility and nitrogen utilization of tropical natural grass hay by goats and sheep. J. Anim. Sci., 43: 879-883.

[60] Ferreira, A.V., L.C. Hoffman, S.J. Schoman and R. Sheridan, 2002. Water intake of Boer goats and Mutton merinos receiving either a low or high energy feedlot diet. Small Rumin. Res., 43: 245-248.

[61] Hadjigeorgiou, I.E., I.J. Gordon and J.A. Milne, 2003. Intake, digestion and selection of roughage with different staple lengths by sheep and goats. Small Rumin. Res., 47: 117-132.

[62] Van, D.T.T., 2006. Some Animal and Feed Factors Affecting Feed Intake, Behaviour and Performance of Small Ruminants doctoral thesis Swedish University of Agricultural Sciences Uppsala .ISSN 1652-6880, ISBN 91: 576-7081-1.

[63] Farid, M.FA., H.M. Abou El-Nasr and N.I. Hassan, 1986. Effect of dietary available carbohydrate level on feed and nitrogen utilization in sheep given urea in the drinking water. World Rev. of animal Prod., 12: 3.

[64] Domingue, B.M.F., D.W. Dellow and T.N. Barry, 1991. Voluntary intake and rumen digestion of lowquality roughage by goats and sheep. J. Agric. Sci., 117: 111-120.

[65] McSweeney, C.S., B. Palmer, R. Bunch and D.O. Krause, 1999. Isolation and characterization of Mediterranean heath woodland range. J. Range Manage, 51: 383-391.

[66] Szumacher-Strabel, M. and A. Cieślak, 2010. Potential of phytofactors to mitigate rumen ammonia and methane production. J. Anim. Feed Sci., 19: 319-337.

[67] Carro, M.D., G. Cantalapiedra-Hijar, M.J. Ranilla and E. Molina-Alcaide, 2012. Urinary excretion of purine derivatives, microbial protein synthesis, nitrogen use, and ruminal fermentation in sheep and goats fed diets of different quality J. Anim. Sci., 90: 3963-3972.

[68] Goel, G. and H.P.S. Makkar, 2012. Methane mitigation from ruminants using tannins and saponins, a status review. Trop. Anim. Health Prod., 44: 729-739.

[69] Aschenbach, J.R., G.B. Penner, F. Stumpff and G. Gabel, 2011. Ruminant nutrition symposium: role of fermentation acid absorption in the regulation of ruminal pH. J. Anim. Sci., 89: 1092-1107.

[70] Brown, D., J.W. Ngambi and D. Norris, 2017. Effect of tanniniferous Acacia karroo leaf meal inclusion level on feed intake, digestibility and live weight gain of goats fed a Setaria verticillata grass hay-based diet Journal of Applied Animal Research (Online) Journal homepage: http://www.tandfonline.com/loi/taar20.

[71]Zarkawi, M., M.R. Al-Masri and K. Khalifa, 2005. Nutritive value of Sesbania aculeate grown on salty soil and its effect on reproductive parameters of Syrian Awassi ewes. Aust. J. of Anim. Res., 56(8): 819-825.

[72] Shiroma, S. and A. Akashi, 1976. Degradation of mimosine in Leucaena leucocephala de Wit by goal rumen microorganisms. Nippon Chikusan Gakkai-ho; 47: 739- 747.

[73] Srivastavam, S.N.L. and K. Sharma, 1998. Response of goats to pelleted diets containing different proportions of sun-dried Leucaena leucocephala. Small Ruminant Research, 28: 139-148.

[74] Fayed, A.M., K.M. Youssef and H.M. Abou-El-Naser, 2001. Nutritional performance of goat fed nonconventional diets based on olive pulp in Sinai. Egyptian: Nutrition and Feeds, 1: 81-89.

[75] Mousa, M.R.M. and H.M. El-Shabrawy, 2003. Growth performance of Damascus kids as affected by feeding system under Semi-arid conditions of North Sinai. J. Agric. Sci. Mansoura Univ, 28(7): 5224-5237.

[76] Mehrez, A.Z., M.R.M. Mousa and H.M. El-Shabrawy, 2011. Effect of feeding varying levels of acacia leaves and twigs on productive performance of Awassi Ewes under Semi-arid conditions of north Sinai. Egyptian J. Nutrition an feeds, 14 (1): 39-52.

[77] Badawy, M.T., H.A. Gawish and A.A. Younis, 2002. Some physiological responses of growing Barki lambs and Baladi kids fed natural desert shrubs. International Symposium on Optimum Resources Utilization in Salt-Affect Ecosystems in Arid and Semi- arid Regions. Cairo, pp: 496-503.

[78] Abdel-Halim, A.M., 2003. Studies of some anti- nutritional factors affecting utilization by ruminants. $\mathrm{Ph}$. D. thesis, Faculty of Science. Ain Shams University.

[79] Shaker, Y.M., S.S. Abou El-Ezz and A.L. Hashem, 2008. Physiological performance of Barki male lambs fed halophytes under semi-arid conditions. J. Agric. Sci. Mansoura Univ., 33 (9): 6393-6408. 
[80] Shaker, Y.M., N.H. Ibrahim, F.E. Younis and H.M. El Shaer, 2014. Effect of Feeding Some Salt Tolerant Fodder Shrubs Mixture on Physiological Performance of Shami Goats in Southern Sinai, Egypt Journal of American Science., 10 (2s): 66-77.

[81] Azamel, A.A., 1997. Physiological responses and daily gain of growing Barki lambs fed on Atriplex halimus, a natural desert shrubs. Egypt. J. Appl. Sci., 12 (2): 1-9.

[82] Tripathy, K.C., B.K. Sahu, N.C. Panda and B.C. Nayak, 1984. Toxicity of tannin acid in goats. Indian J. Anim. Sci., 54 (11): 1091-1093.

[83] Bravo, L., E. Manas and F.S. Calixto, 1993. Dietary non extractable condensed tannins as indigestible compound: Effect on fecal weight and protein and fat excretion. J. Sci. Food Agric., 63: 63-68.

[84] Romero, M.J., J. Madrid, F. Hernandez and J.J. Ceron, 2000. Digestibility and voluntary intake of Vine leaves (Vitis vinifera L.) by sheep. Small Ruminant Res., 38: 191-195. 